\title{
Model-based fault detection for proton exchange membrane fuel cell systems
}

\author{
Mahanijah Md Kamal*, Dingli Yu \\ "Control System Research Group, School of Engineering, Liverpool John Moores University, Byrom Street, Liverpool L3 3AF, UNITED KINGDOM \\ "Corresponding Author: e-mail: M.B.Md-Kamal@2010.ljmu.ac.uk
}

\begin{abstract}
In this paper, an intelligent model-based fault detection (FD) is developed for proton exchange membrane fuel cell (PEMFC) dynamic systems using an independent radial basis function (RBF) networks. The novelty is that this RBF networks is used to model the PEMFC dynamic systems and residuals are generated based on the differences between the PEMFC systems and RBF networks model. Later, based on this information the RBF model performed the FD including identification and classification. Five types of faults have been introduced to the PEMFC dynamic systems which occurred in the actuator, component and three sensors part. By considering the faults in the PEMFC systems is $10 \%$ changes in each component, the efficiency of the proposed model is studied. The developed model is tested on MATLAB/Simulink simulation. The results show that all the simulated faults can clearly be detected and classified by this intelligent model-based FD. By using this intelligent FD method, the PEMFC dynamic systems is intelligent to detect the faults quickly, classify them and then appropriate action can be taken immediately.
\end{abstract}

Keywords: Proton Exchange Membrane Fuel Cell, model-based, RBF networks, fault detection, MATLAB/Simulink

DOI: http://dx.doi.org/10.4314/ijest.v3i9.1

\section{Introduction}

Process faults, if undetected, have a serious impact on process economy, product quality, safety, productivity and pollution level. In order to detect, diagnose and correct these abnormal process behaviors, efficient and advanced automated diagnostic systems are of great importance to modern industries (Albert et al, 2007). Once a fault has been detected and its evolution is monitored, the severity of that fault can be evaluated and a decision can be made on the course of action to be taken. Monitoring creates the opportunity to strategically plan and schedule outrages and to manage equipment utilization and availability (Jean-Pierre and Joseph, 1995). A variety of fault detection and diagnosis techniques have been developed and published in the area of diagnostic problem in the PEMFC dynamic systems. These techniques include model-based approaches, knowledge based approaches, qualitative simulation based approaches, neural network based approaches and classical multivariate statistical techniques. For fault detection and diagnosis problem, the most effective way will be model-based approach based on a decision-making where a limit or threshold of a residual is generated. Here, the difference between the actual and the estimated output is used as a residual vector. In the aspect of hydrogen safety and efficiency for PEMFC, Ari et al. (2005) has developed a computer simulation tool which can be used to detect and monitor faults in the hydrogen stations. While Salim and Lecoeuche (2009) proposed a modelbased observer deals with water management issue which can affect the cell performance. (Teresa et al., 2009) used this method based on the relative residual fault sensitivity by checking the consistency of observed behaviour while fault isolation tries to isolate the component that is in fault in different sensitivities. In order to tackle the nonlinear systems behavior, the used of neural network has been used widely in the FD for fuel cell systems. Considering with the flooding issue, (Luis et al, 2006) have used the Bayesian network for fault diagnosis with the probabilistic approaches such as $\mathrm{K} 2$ and MCMC algorithm. While (Yousfi et al, 2010) used elman neural network (ENN) for fault diagnosis in the PEMFC systems to overcome the same problem. Later, (Roy et al, 2008) also used the Bayesian network as a supervisor system which was able to diagnose degradation and catastrophic faults in the equipment. 
From the observation, even though some of the mentioned methods dealing with FD but there is still a lack of information on FD especially for classification. Most of the existing method for FD shows the response of fault individually instead of multiple faults all at once. Besides that, some of the work is focused more on mathematical modeling approach to do FD. Therefore, based on the previous researcher's work and also concerning about the cost, safety, efficiency and the reliability of the PEMFC performance, the best way to implement the FD technique is through the development of model-based diagnosis based on residual fault sensitivity generation. Model-based approach gives the insight analysis of the subsystem interactions and also provides guidelines during the systems operation. The system behavior can be analyzed for depth understanding and this information can be used for future design and development of the systems. The used of neural network will overcome the nonlinear systems behavior of PEMFC dynamic systems. Therefore, the contribution of this research is to develop an intelligent FD scheme for PEMFC dynamic systems using a model-based RBF networks. This develop model is not only can detect the five faults in the PEMFC systems but it is also can classify the type of faults efficiently.

\section{PEMFC Systems}

Nowadays, there is a great demand and interest in the renewable energy technology which has provided motivation for researchers to perform research especially in fuel cell. Fuel cell research had received a great deal of attention because of its strategic importance in global energy conversion and also its positive impact towards the environment. Due to high electrical efficiency, flexibility with respect to power and capacity, long lifetime and no pollutions, the fuel cells are rapidly becoming significant source of power in the future. Among fuel cell technologies, the proton exchange membrane has received a lot of attention. PEMFC is based on hydrogen technology and operates at low temperatures between the ranges of $60^{\circ}-100^{\circ}$. Besides that, the PEMFC also has a 50\% electrical efficiency. Therefore, these characteristic allow the use of PEMFC in many applications such as for transportation, telecommunication, portable utilities, stationary and distributed power generation.

A fuel cell stack (FCS) cells need to be integrated with several auxiliary components to form a complete fuel cell systems. In this research, the PEMFC simulator model developed by Jay et al. (2004a) is used as a case study for FD. The diagram in Figure 1 shows an example of fuel cell systems which is augmented by four auxiliary system; hydrogen supply systems, air supply system, cooling system and humidification system. The air is supplied by an air compressor, which is used to increase the power density of the overall system. The air supply consists of an air compressor, an electric motor and manifolds between the components. The compressor not only achieves desired air flow but also increases air pressure which significantly improves the reaction rate at membrane, thus the overall efficiency and power density (Jay et al, 2004b).

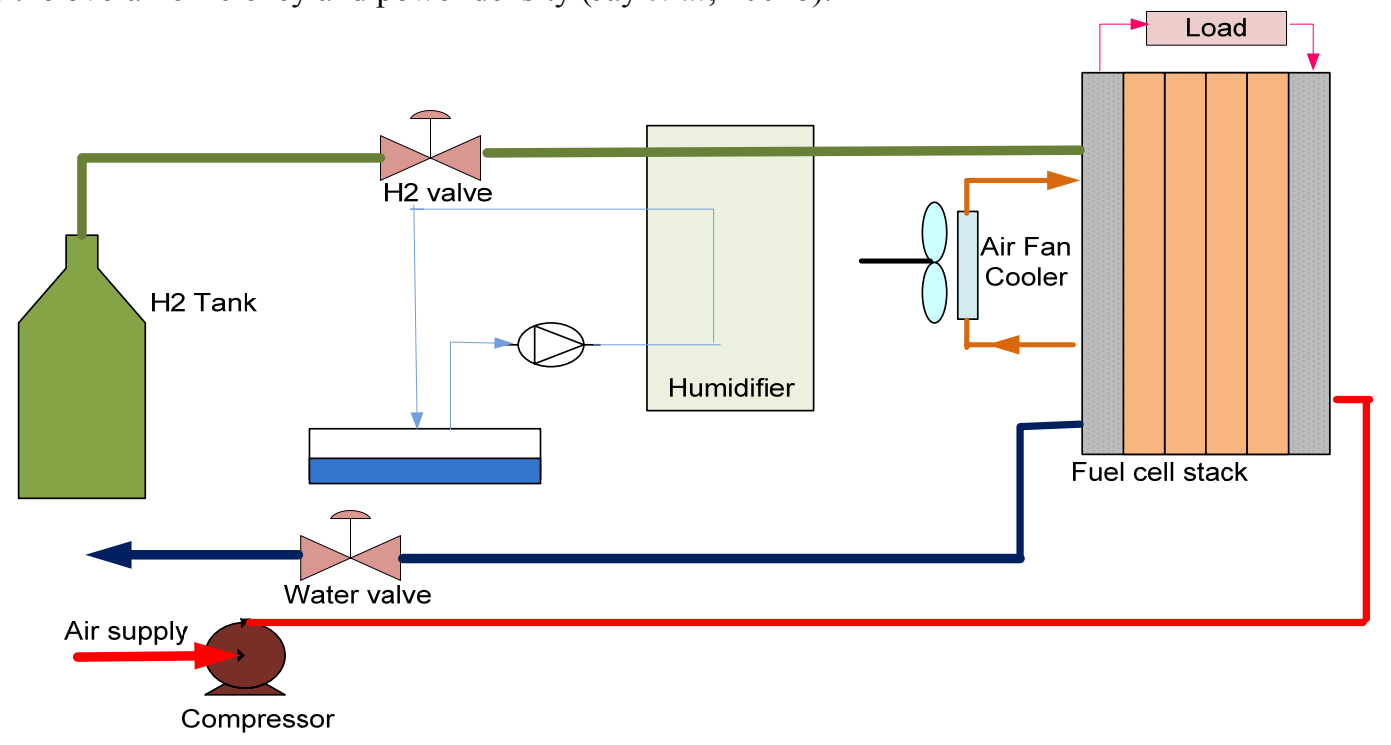

Figure 1: The diagram of FC system proposed by (Jay et al, 2004a)

\section{Radial Basis Function Networks}

A neural network provides a general way to model a nonlinear system with memory and it has been used by many researchers to describe the relationship between the input and output of monitored systems. Radial basis function (RBF) neural networks is a forward network consist of three layers which are the input layer, hidden layer and output layer. This RBF neural networks is chosen because of its characteristic which has the ability to approximation of a nonlinear input system to a linear output. Besides that the training process is faster and better compared with another neural network. The RBF networks is capable of approximating any continuous function with certain precision level and therefore, can be used in dynamic system modeling and control (Mingyu 
et al., 2009). RBF networks used a k-means clustering algorithm which the incoming weights from the input layer become centre of clusters of input vectors. The k-means clustering algorithm determines the closest centre of the RBF networks. Every RBF output is multiplied by the corresponding linear weight and all are summed up as the RBF networks output. The most popular RBF is a Gaussian type that is characterized by a centers $c_{i}$ and a weights $w_{i}$. The centers $c_{i}$ and weights $\mathrm{w}_{\mathrm{i}}$ are complex values whereas the output from the hidden layer is real valued. The input-output mapping for the RBF network (Mingyu et al, 2009) can be described as:

$$
y(x)=\sum_{i=1}^{K} w_{i} \phi_{i}(x)
$$

where the approximating function $\mathrm{y}(\mathrm{x})$ is represented as a sum of $\mathrm{K}$ radial basis functions, each associated with a different center $\mathrm{c}_{\mathrm{i}}$ and weighted by an appropriate coefficient $\mathrm{w}_{\mathrm{i}}$. Among RBF, $\varphi_{\mathrm{i}}(\mathrm{x})$ is the Gaussian function (Mingyu et al, 2009) :

$$
\varphi_{i}(x)=\exp \left(\frac{\left\|x-c_{i}\right\|^{2}}{\rho^{2}}\right)
$$

where $c_{i}$ is the radial basis centre, $\rho$ is the variance of the Gaussian function, and $\mathrm{x}$ is the input vector.

In this work, for training and testing purposes, the constructed structure of RBF networks used consist of two input layers $(\mathrm{x}=2)$, twenty-two hidden layers $(\mathrm{nh}=22)$ and three output layers $(\mathrm{y}=3)$. The structure of $\mathrm{RBF}$ networks implemented in this work is presented in Figure 2.

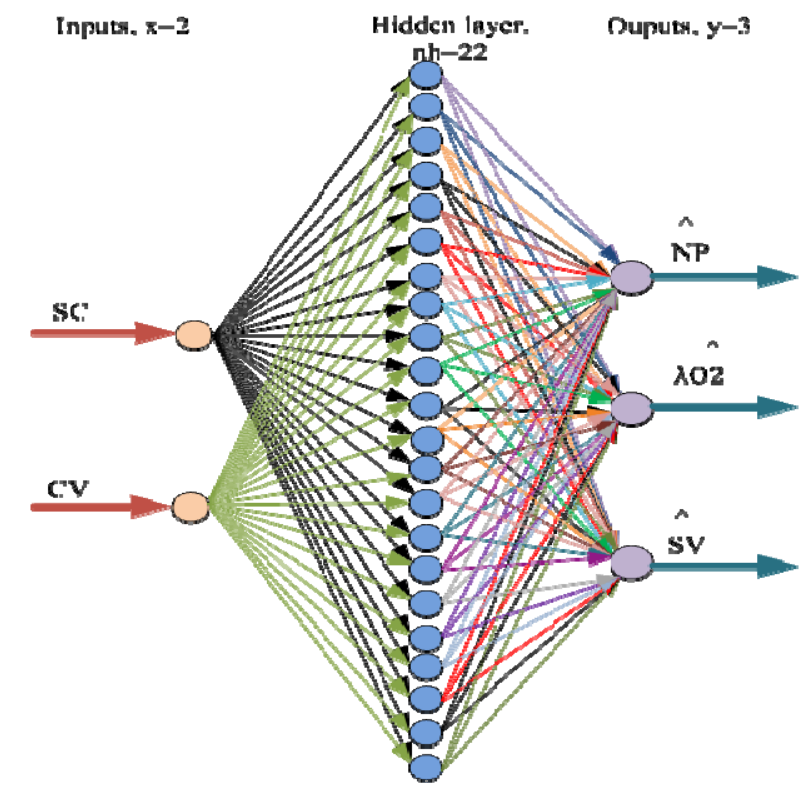

Figure 2: The structure of RBF networks with two inputs and three outputs

The training session of the RBF network uses the error in the output values to update the weights connecting the layers, until a minimize error is achieved. Training the network is a matter of determine the weights and minimize the generated mean absolute error (MAE) (Zhai et al, 2007) defined by the following equation:

$$
M A E=\frac{1}{N} \sum_{k=1}^{N}|e(k)-\hat{e}(k)|
$$

where $\mathrm{e}(\mathrm{k})$ is the true value and $\mathrm{e}(\mathrm{k})$ is the estimated value.

\section{Fault Detection}

Early detection of faults inside the PEMFC systems can extend the life of the FCS. The most effective method of detecting a fault in PEMFC systems is by observing the volume of the air flow in the compressor, the pressure in the cathode and sensors installed inside the systems. Normally the overall efficiency and power density of FCS is indicated by oxygen excess ratio $\lambda \mathrm{O}_{2}$. Low values of $\lambda \mathrm{O}_{2}$ indicates low oxygen concentration in the cathode or oxygen starvation and high oxygen excess ratio, and thus high oxygen partial pressure, improves the power of the stack. The oxygen excess ratio must be regulated to $\lambda \mathrm{O}_{2} \geq 1$ to prevent the starvation phenomenon (Carlos et al, 2010). In (Jay et al, 2004b), the authors propose $\lambda \mathrm{O}_{2}=2$ because this value prevents oxygen 
starvation effect and ensure a high efficiency in its experimental system. Therefore, FD in the auxiliary systems need to make fine and fast adjustments to satisfy performance, safety and reliability standards that are independent of age and operating conditions of FCS (Jay et al., 2004c) .

In this work, the PEMFC dynamic systems simulator developed in (Jay et al, 2004a) has been modified in order to introduce five types of faults that might occurs in the PEMFC dynamic systems. To design FD scheme, dynamic neural network of RBF has been developed in this paper using the MATLAB/Simulink environment. To monitor and implement the FD technique, a model-based FD model is employed to describe the nominal behavior of the PEMFC systems. The technological process can be treated as a system which consists of some major types of subsystems such as actuator, process and sensors. In order to ensure that the RBF model used for FD in this research is correct, the online PEMFC simulator developed by Jay et al. (2004a) has been used. Here, the online simulation of PEMFC systems without any faults occur in the system has been conducted to see the corresponding outputs measurements. Later, this developed model has been modified in order to introduce five possible faults which might occur during the operations of PEMFC. Figure 3 shows the five types of faults used in this work.

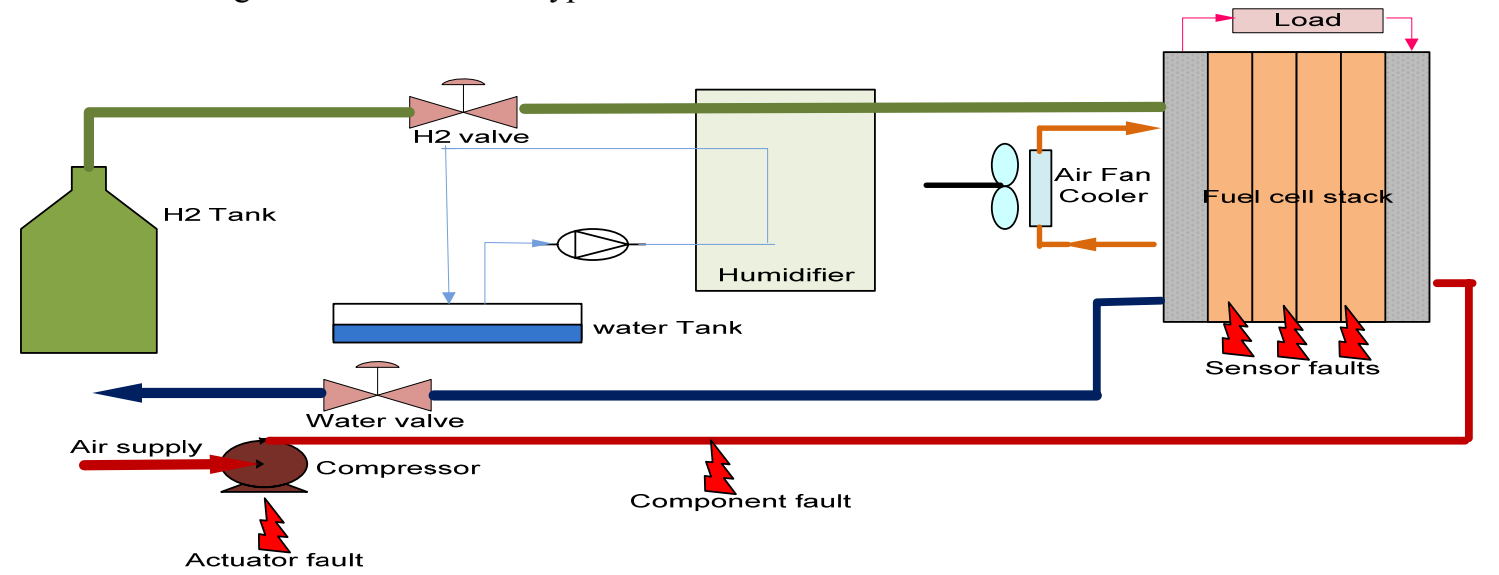

Figure 3: Five faults introduced to the PEMFC system proposed by (Jay et al, 2004a)

Failure strategies used in this work are being introduced in the actuator, component and sensors in the PEMFC systems. The faults occur in the system is introduced as a 10\% drop of the air flow in the actuator, a leak of the pressure in the manifold and also faults in the sensor devices. The integrated model coded in MATLAB-Simulink shown in Figure 4 is used for FD. The stack current $\mathrm{SC}$ and the compressor motor voltage $\mathrm{CV}$ are the inputs. Leak is the measured disturbances while net power NP, oxygen excess ratio $\lambda \mathrm{O}_{2}$ and stack voltage $\mathrm{SV}$ are the output variables. Again, the RBF networks is trained and tested but this time, the inputs to the RBF networks is a faulty data occurs during the operation of PEMFC systems.

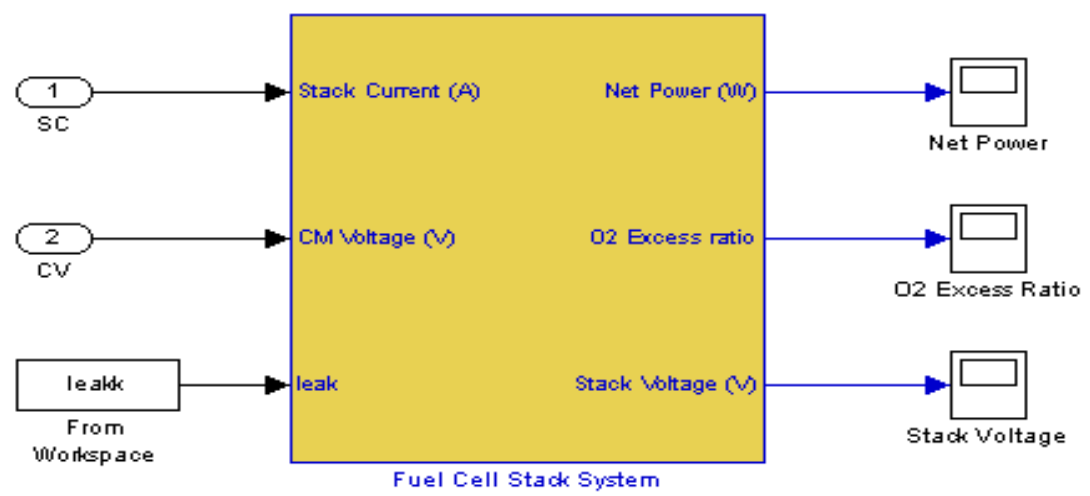

Figure 4: The MATLAB/Simulink with leak

The aim of detection system is to detect the faults in each subsystem and provide the information about their size and sources. One of the most known FD methods is technique based on residual generation (Roozbeh et al., 2009). FD can be performed of a three step algorithm. Firstly, one or several signals reflecting faults in the process behavior are generated. These signals are called residuals. In the second steps, the residuals are evaluated. A decision is made using these residuals, in order to determine the time and the location of possible faults. Finally, the nature and likely cause of the faults are analyzed by the relations between the symptoms and their physical causes. The block diagram of FD used in this work is shown in Figure 5. 


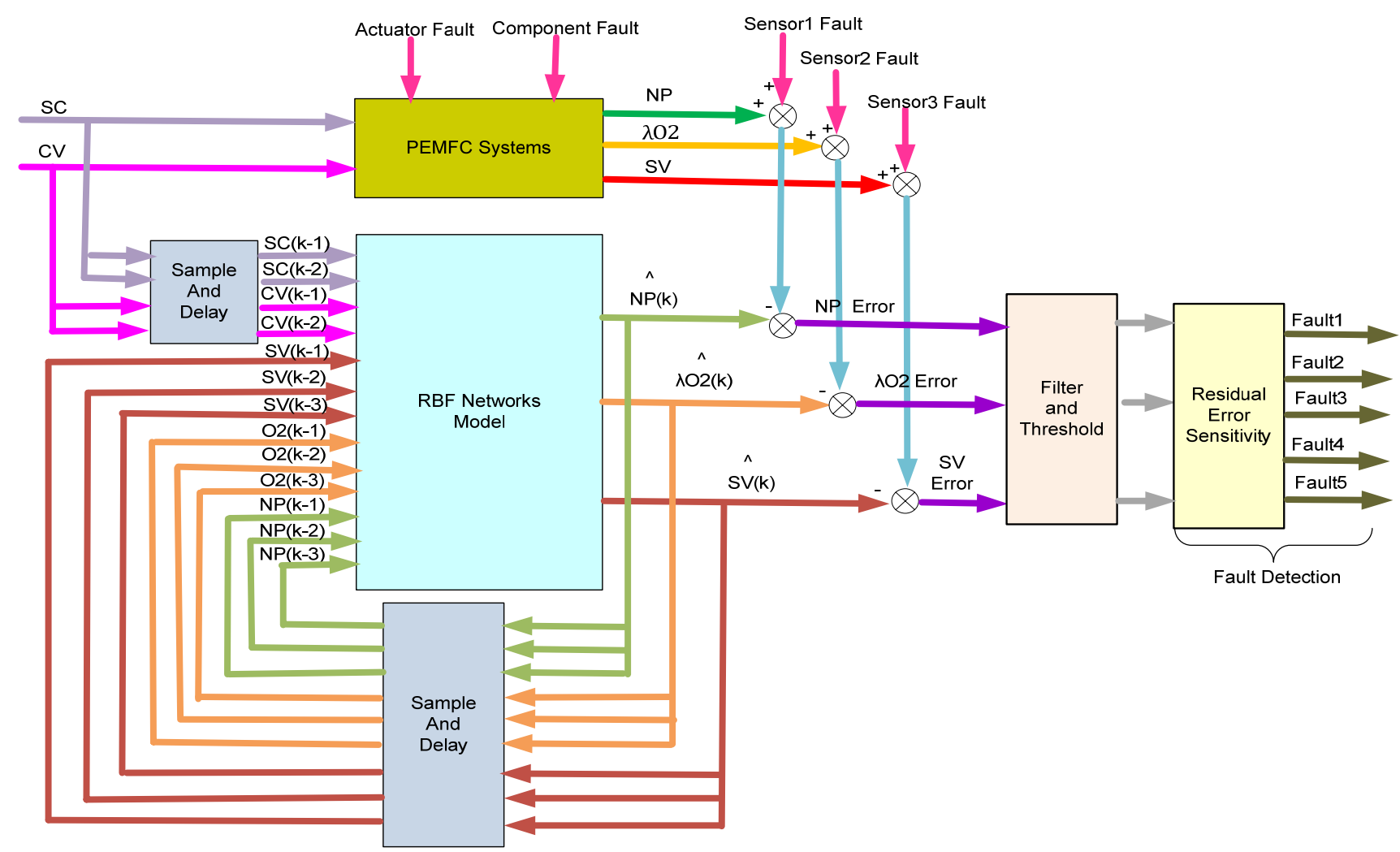

Figure 5: The block diagram of fault detection algorithm used in the PEMFC systems

At initial stage, a set of random amplitude signals (RAS) excitation signals has been injected as inputs for the stack current SC and compressor motor voltage CV of the PEMFC systems. These signals ranging from 100 to 300 amperes for the stack current and from 100 to 235 volts for the compressor motor voltage. The matrix form of the inputs and outputs can be shown as follows:

$$
\begin{aligned}
& u=\left[\begin{array}{cccc}
S C_{1} & S C_{2} & \cdots & S C_{6000} \\
C V_{1} & C V_{2} & \cdots & C V_{6000}
\end{array}\right] \\
& y=\left[\begin{array}{cccc}
Y_{N P 1} & Y_{N P 2} & \cdots & Y_{N P 6000} \\
Y_{O 21} & Y_{O 2} & \cdots & Y_{O 26000} \\
Y_{S V 1} & Y_{S V 2} & \cdots & Y_{S V 6000}
\end{array}\right]
\end{aligned}
$$

where $u$ and $y$ is the input and output of the PEMFC systems respectively.
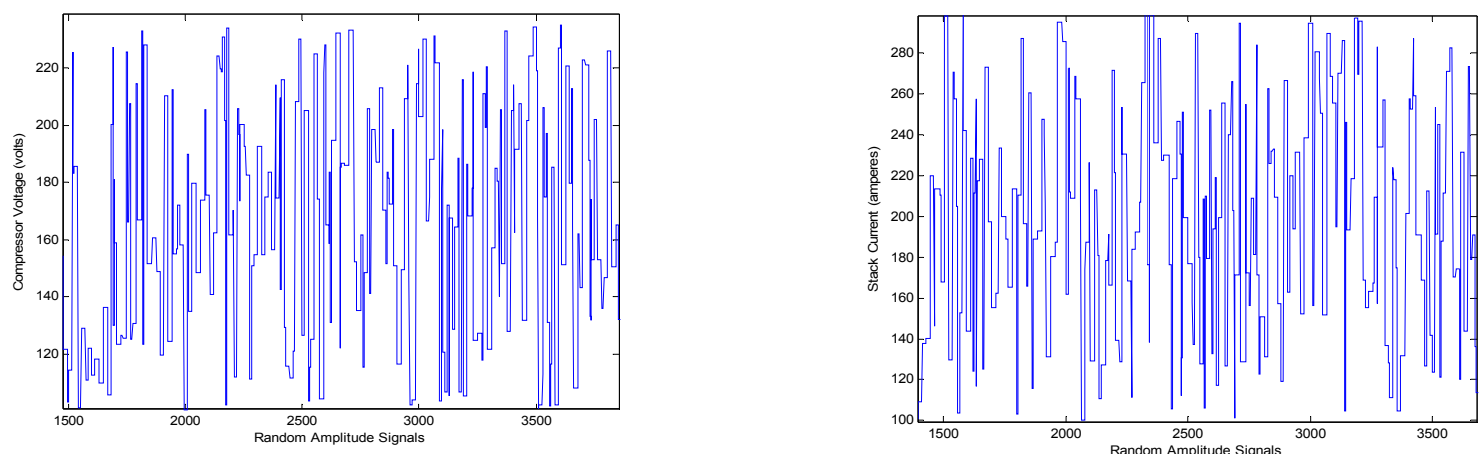

Figure 6: The excitation signals for compressor voltage and stack current 
Figure 6 shows the example of RAS excitation signals used in this work. The signals are generated randomly by the proposed model to cover all range of frequencies. Once the RAS have been generated, these signals are used as inputs data for training and testing purposes in the RBF algorithm. In order to ensure the convergence of the RBF networks, the input and the output signals need to be normalized in the range of zero to one. These signals are fed into the RBF using the scaled value where:

$$
\begin{gathered}
\text { scaled_input }=\frac{\text { actual_input }- \text { actual_input } t_{\min }}{\text { actual_input } t_{\max }-\text { actual_input }{ }_{\min }} \\
\text { scaled_output }=\frac{\text { actual_output }- \text { actual_output }{ }_{\min }}{\text { actual_output }{ }_{\max }-\text { actual_output } t_{\min }}
\end{gathered}
$$

The new scaled value of inputs and outputs signals had been divided into two categories. The first 5000 data samples are used as training sets and another 1000 data samples are used for testing purposes. At this stage, the RBF networks is trained with a set of free faults data and serves as the reference basis to the diagnosis procedure. After several training, the optimum value from the training process is used in the testing process.

Next step, to generate the residual evaluation that indicate differences between the RBF networks model's output and the PEMFC measured process output. The free fault and faulty training data sequences are fed to the input layer randomly after all the weights and the thresholds are initialized with random numbers. A threshold needs to be set to evaluate whether or not the residuals are under faulty conditions. By doing this, from the simulation, it determine whether the outputs of PEMFC system consists a faulty data measurements or not. The threshold test used in this work is set at one. However, from the observation and after the trials and errors, the values of threshold are found between the ranges of $0.6 \times 10^{-3}-10 \times 10^{-3}$. Residuals are zero under an ideal condition, therefore the residual signal $\mathrm{r}(\mathrm{t})$ is set with a threshold function $\mathrm{T}(\mathrm{t})$ according to equation (12) to indicates the fault signals $\mathrm{f}(\mathrm{t})$ of PEMFC systems.

$$
\text { Residual }=\left\{\begin{array}{lll}
r(t) \leq T(t) & \text { for } & f(t)=0 \\
r(t)>T(t) & \text { for } & f(t) \neq 0
\end{array}\right.
$$

The residual signal $r(t)$ is calculated as

$$
r=y-\hat{y}
$$

where $y$ is the PEMFC plant measurements and $\hat{y}$ is the RBF networks model prediction.

The residual signals produce after the simulation is still influence by distortions and noises. Therefore, to improve the accuracy of the model, and to reduce the noises, the residual signals need to be filtered digitally. For data analysis, a Bessel filter is used to reduce the noises in the signals. The Bessel filter had the benefit of having a linear phase shift. Later, the residual error sensitivity is calculated to perform the FD. The residual errors sensitivity is given by:

$$
r e=\sqrt{\frac{\left(e_{N P}^{2}+e_{\lambda O 2}{ }^{2}+e_{S V}{ }^{2}\right)}{6.25}}
$$

Where $e_{\mathrm{NP}}$ is the error vector of net power, $\mathrm{e}_{\lambda \mathrm{O} 2}$ is the error vector of the oxygen excess ratio and $\mathrm{e}_{\mathrm{SV}}$ is the error vector of stack voltage. Once the RBF networks detected the faults, it discovers the type of faults occurred based on hardware malfunction in the PEMFC plant. With respect to the whole training and test set, a threshold value for residual errors sensitivity has been defined. It can be said that the range of threshold for residual errors sensitivity is between $0.5 \times 10^{-3}-1 \times 10^{-3}$.

\section{Simulation results}

In order to validate the RBF networks model, the net power, the oxygen excess ratio and stack voltage obtained from the simulation are compared with measured results of the PEMFC systems. In this PEMFC plant, five types of faults are considered. In this simulation, 6000 samples have been used for FD using two types of samples named as Samples1 and Samples2. For both samples, the faults being introduced are considered as $10 \%$ change in the PEMFC systems. Here, the sampling time, $\mathrm{t}_{\mathrm{s}}$ used is set at $20 \mathrm{~ms}$ with hidden layer at 22 nodes. Data samples used for both simulation is 6000 data samples. Table 1 summarized the types of fault introduced to the PEMFC systems with the range of the faults occurred. 
Table 1: The faults data used in the PEMFC systems

\begin{tabular}{|c|c|c|c|}
\hline \multirow{2}{*}{ Item } & Types of fault & No. of data & Data range \\
\hline \multirow{4}{*}{ Samples1 } & Sensor1 & 200 & $1000-1200$ \\
\cline { 2 - 4 } & Sensor2 & 200 & $2000-2200$ \\
\cline { 2 - 4 } & Sensor3 & 200 & $3000-3200$ \\
\cline { 2 - 4 } & Component & 200 & $4000-4200$ \\
\cline { 2 - 4 } & Actuator & 200 & $5000-5200$ \\
\hline \multirow{5}{*}{ Samples2 } & Component & 200 & $1000-1200$ \\
\cline { 2 - 4 } & Actuator & 200 & $2000-2200$ \\
\cline { 2 - 4 } & Sensor1 & 200 & $3000-3200$ \\
\cline { 2 - 4 } & Sensor2 & 200 & $4000-4200$ \\
\cline { 2 - 4 } & Sensor3 & 200 & $5000-5200$ \\
\hline
\end{tabular}
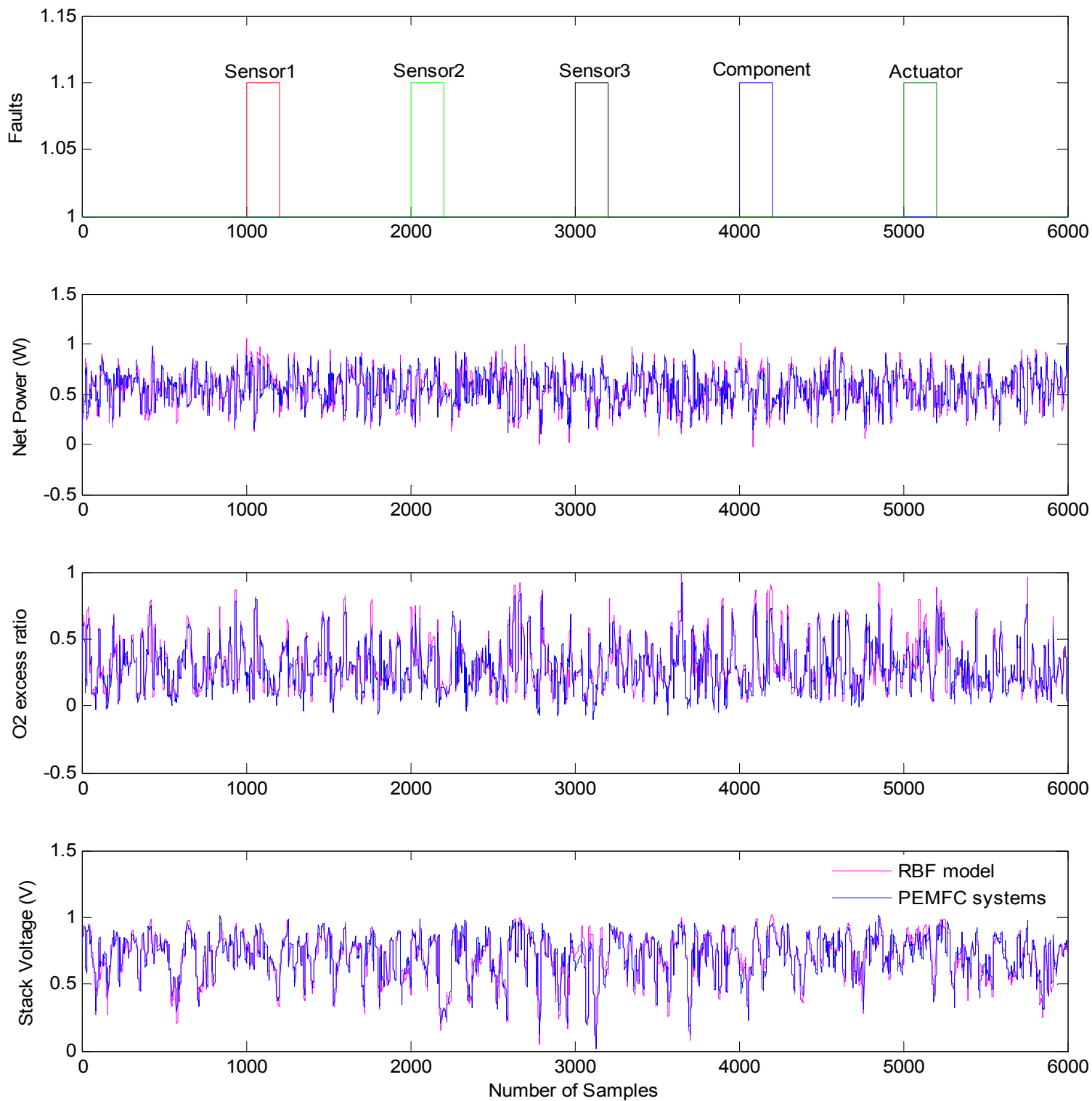

Figure 7: The actual and estimated output of $\mathrm{NP}, \lambda \mathrm{O}_{2}$ and $\mathrm{SV}$ during testing process for Samples1 data set 
Figure 7 shows the output simulation of $\mathrm{NP}, \lambda \mathrm{O}_{2}$ and $\mathrm{SV}$ when the faults occur in the systems for testing data set. Here, there are not many differences between the measured outputs and the estimated outputs. However, there is a gap and differences between these two signals especially at the introduction of faults based on Table 1.
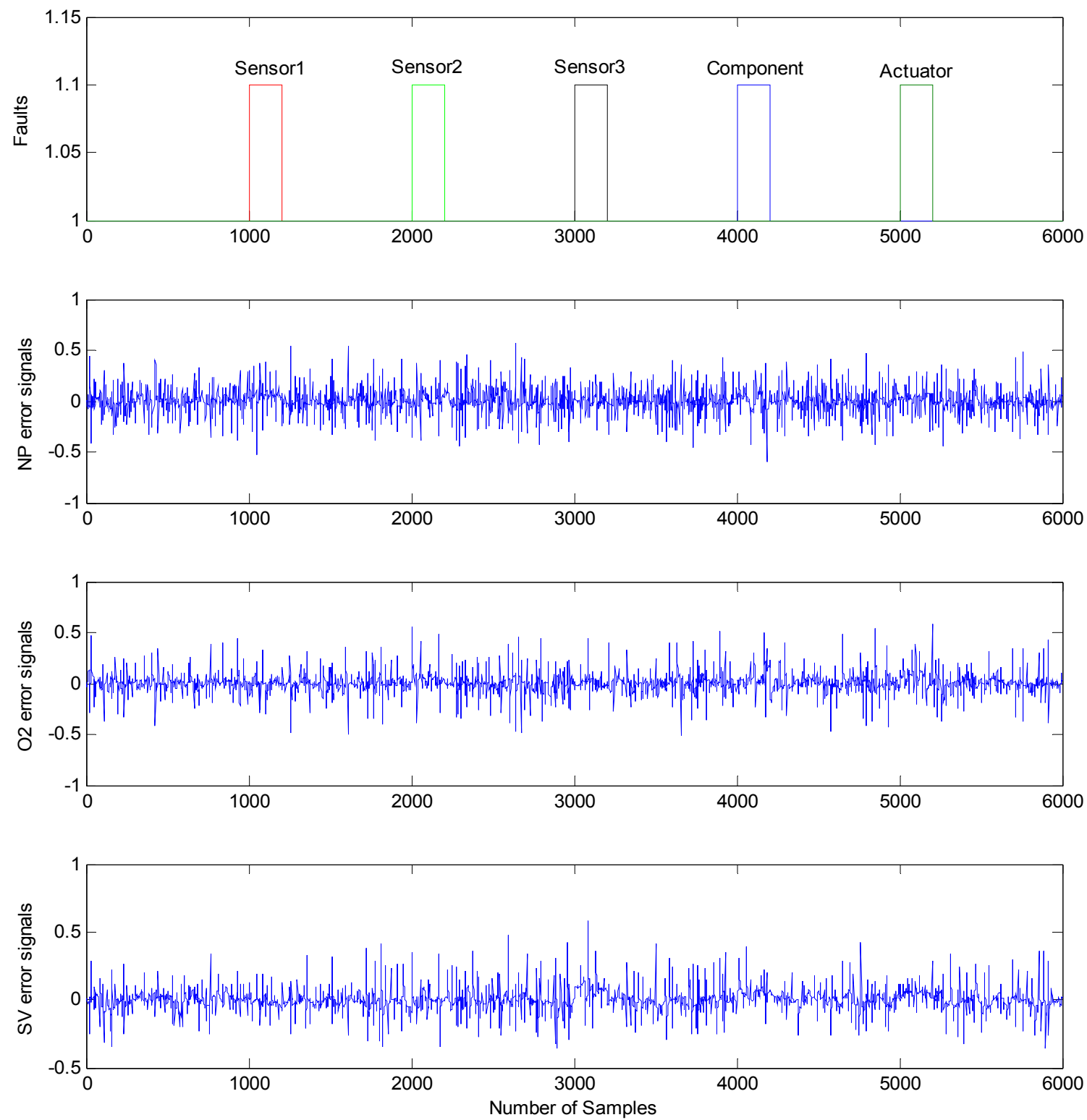

Figure 8: The error signals of NP, $\lambda \mathrm{O}_{2}$ and $\mathrm{SV}$ between the PEMFC Systems and RBFNN for Samples1

In Figure 8, it shows the error signals of the output NP, $\lambda \mathrm{O}_{2}$ and $\mathrm{SV}$ with the existing of faults in the signals. These output signals need to be filtered before the FD can be applied due to the influence of noise inside the signals. Based on the result obtained, there is a clear phase shift at samples number 3000 and 5000 at the output of SV error signals. However, the fault signals in the output of NP and $\lambda \mathrm{O}_{2}$ cannot be seen clearly. 

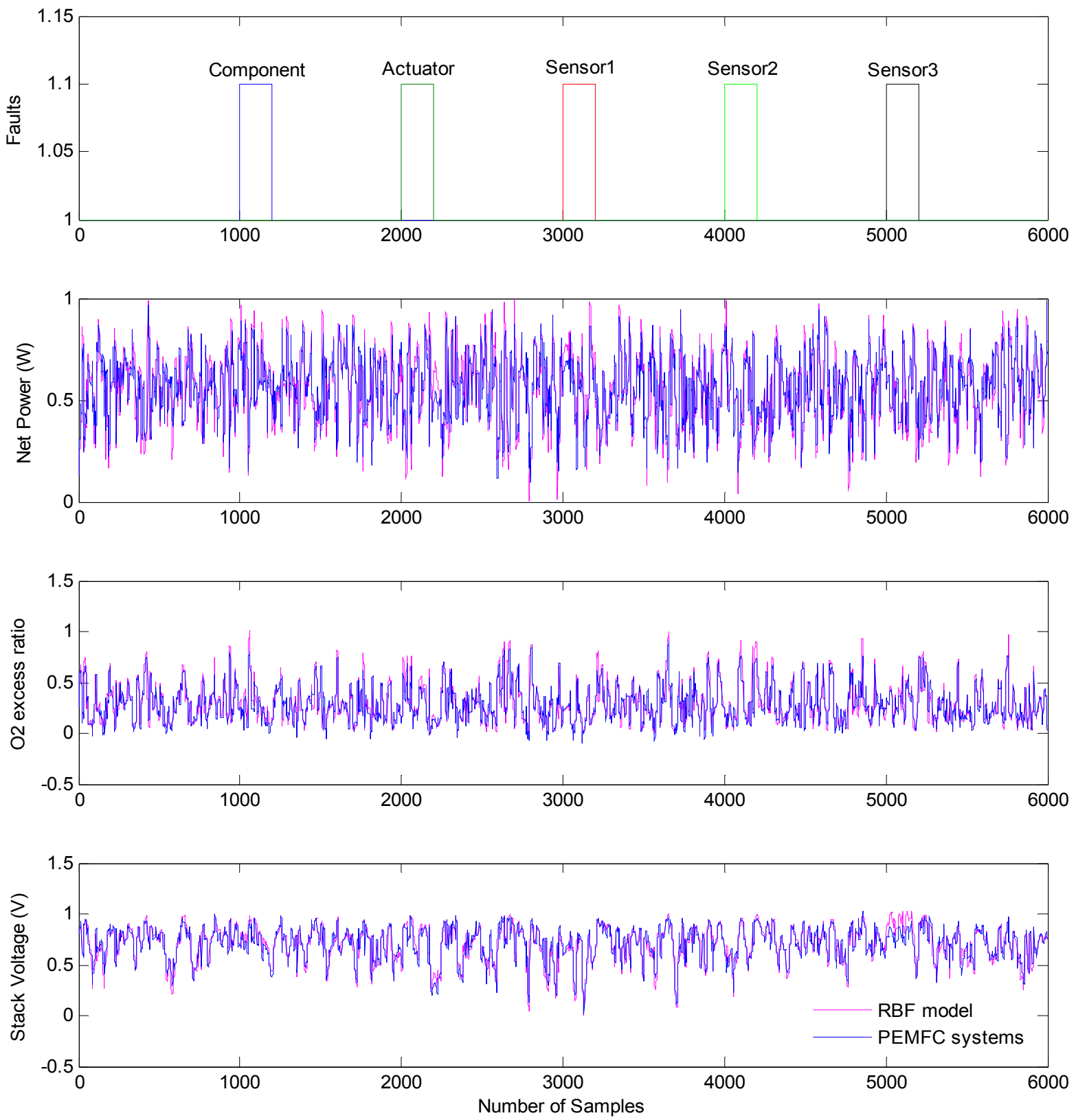

Figure 9: The actual and estimated output of $\mathrm{NP}, \lambda \mathrm{O}_{2}$ and $\mathrm{SV}$ during testing process for Samples2 data set

Figure 9 shows the output simulation of $\mathrm{NP}, \lambda \mathrm{O}_{2}$ and $\mathrm{SV}$ when the faults occur in the systems for testing data set. Again, there are not many differences between the measured outputs and the estimated outputs. However, there still a gap and differences between these two signals especially at the introduction of faults based on Table 1. 

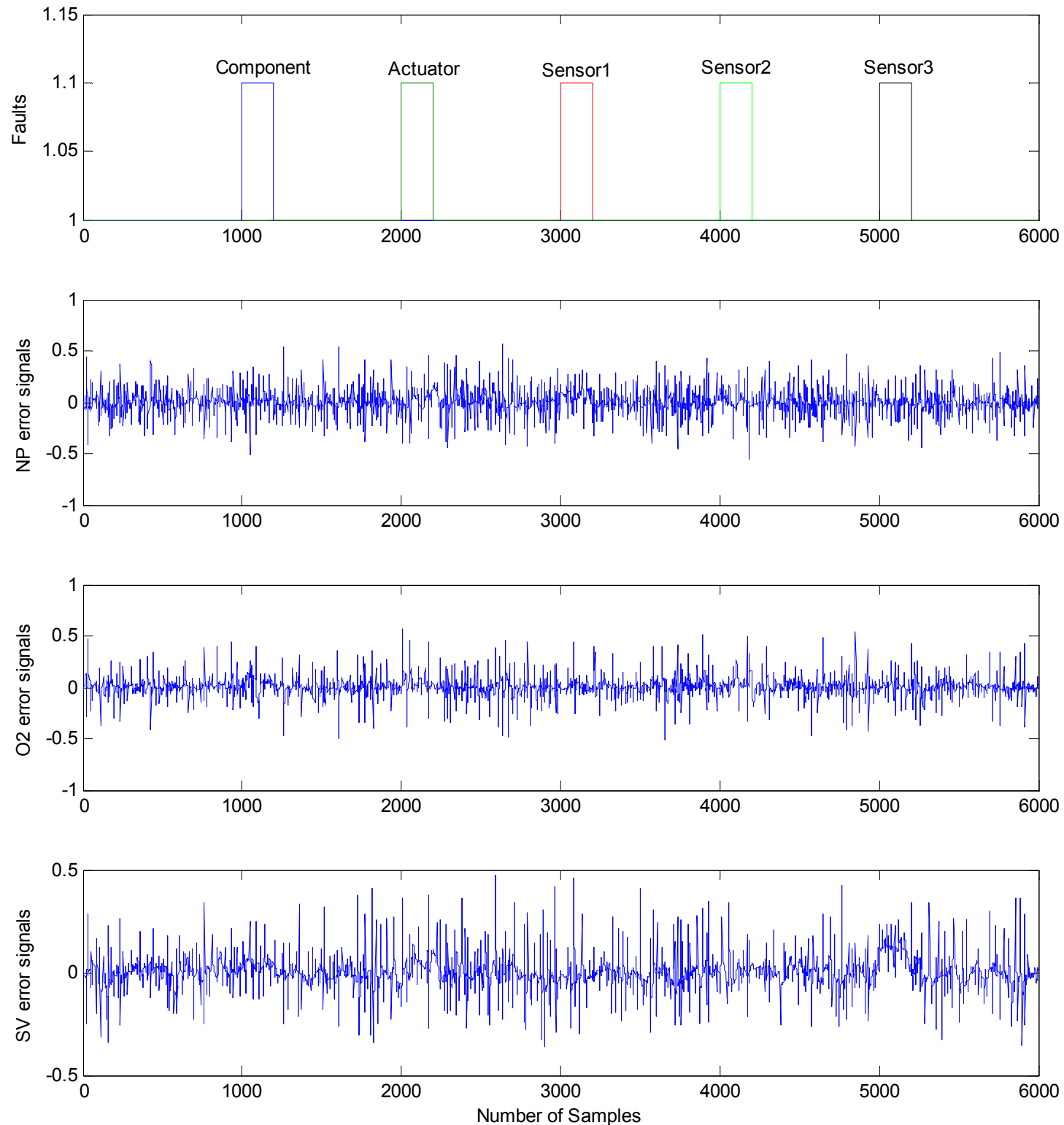

Figure 10: The error of $\mathrm{NP}, \lambda \mathrm{O}_{2}$ and $\mathrm{SV}$ between the PEMFC Systems and RBFNN for Samples2

In Figure 10, it shows the error signals of the output NP, $\lambda \mathrm{O}_{2}$ and $\mathrm{SV}$ with the existing of faults in the signals. Based on the result obtained, there is a clear phase shift at samples number 5000 at the output of SV error. Again, there are no differences in the output of $\lambda \mathrm{O}_{2}$ and SV. Therefore, these output signals need to be filtered before the FD can be applied due to the influence of noise inside the signals. 

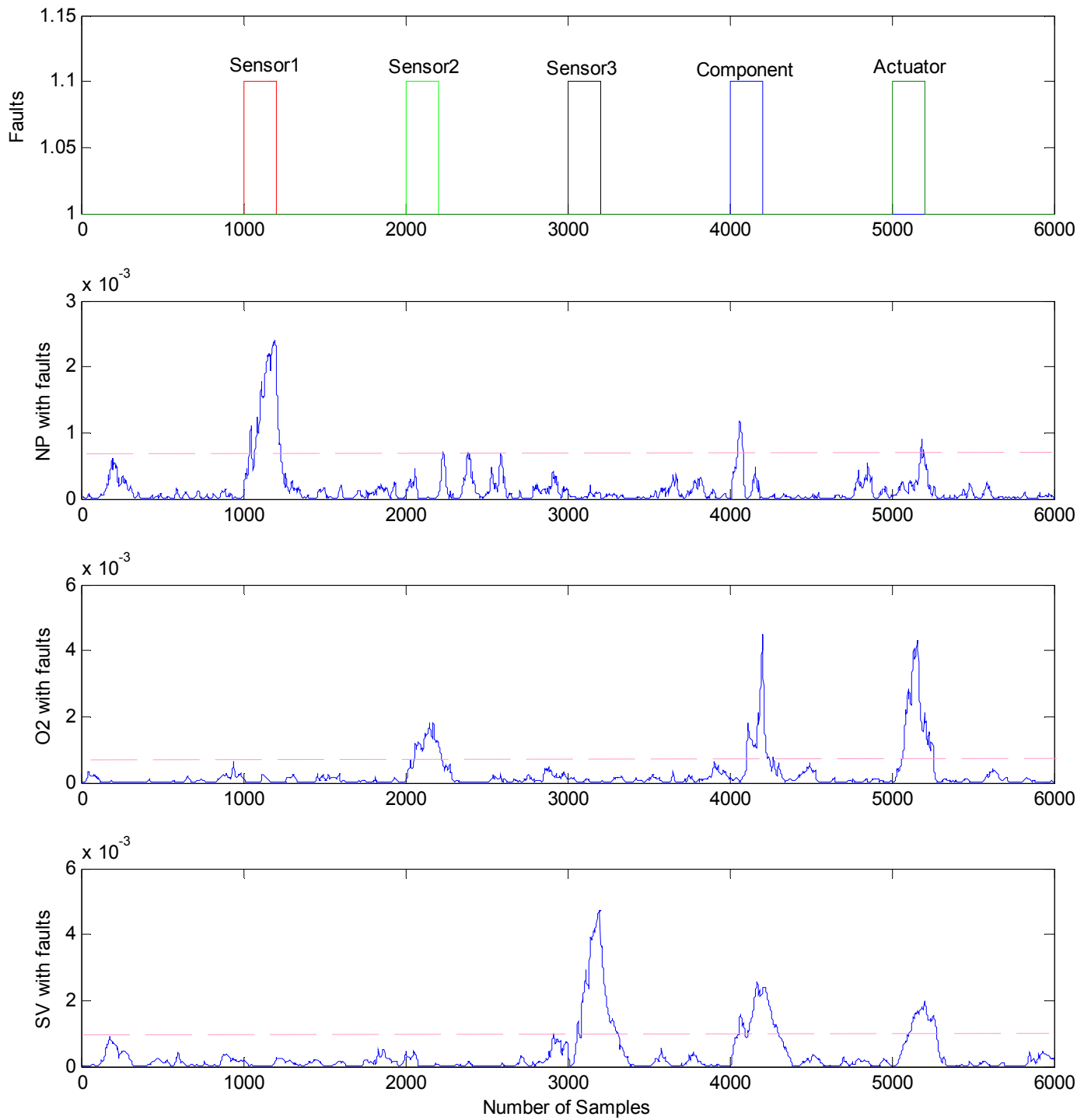

Figure 11: The filtered signals of $\mathrm{NP}, \lambda \mathrm{O}_{2}$ and $\mathrm{SV}$ and respective threshold for Samples1

Figure 11 shows the filtered signals of faults at the outputs plant of Samples1 before the FD can be applied. Here, the faults occurrence can be clearly identified and detected with their respective threshold. From the results obtained, there are two possible faults in NP output signal due to sensorl fault and component fault. Faults also have occurred in the $\lambda \mathrm{O}_{2}$ output signal due to sensor2, component and actuator faults. There are also three types of faults in SV output signal due to sensor3, component and actuator faults. 

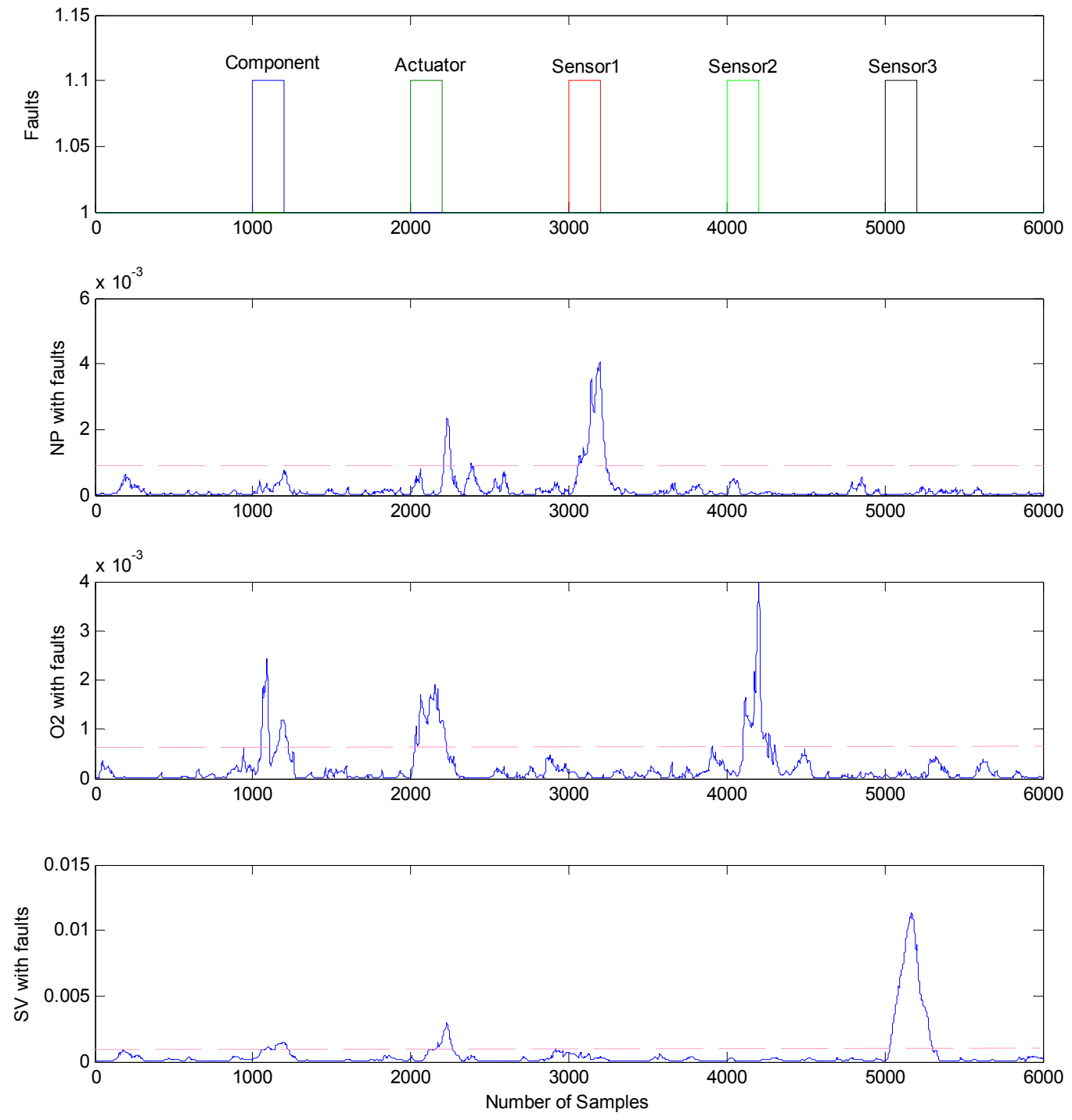

Figure 12: The filtered signals of $\mathrm{NP}, \lambda \mathrm{O}_{2}$ and $\mathrm{SV}$ and respective threshold for Samples2

Figure 12 shows the filtered signals of faults at the outputs plant of Samples2. From the results it shows that there are faults in NP output signal due to actuator and sensorl faults, while in $\lambda \mathrm{O}_{2}$ there are three types of faults produced by component, actuator and sensor2 faults. But in SV output signal there is a clear fault due to sensor3 malfunction in the systems. It is also an actuator fault in the SV output signal.

Table 2: The value of MAE for Samples 1 and Samples 2

\begin{tabular}{|c|c|c|c|c|c|c|}
\hline \multirow{2}{*}{ Task } & \multicolumn{3}{|c|}{ Samples 1 } & \multicolumn{3}{c|}{ Samples 2 } \\
\cline { 2 - 7 } & $\begin{array}{c}\text { Net Power } \\
(\mathrm{W})\end{array}$ & $\lambda \mathrm{O}_{2}$ & $\begin{array}{c}\text { Stack } \\
\text { voltage (V) }\end{array}$ & $\begin{array}{c}\text { Net Power } \\
(\mathrm{W})\end{array}$ & $\lambda \mathrm{O}_{2}$ & $\begin{array}{c}\text { Stack } \\
\text { voltage (V) }\end{array}$ \\
\hline $\begin{array}{c}\text { Training \& Testing } \\
\text { (Free fault) }\end{array}$ & 0.0061 & 0.0048 & 0.0035 & 0.0061 & 0.0048 & 0.0035 \\
\hline $\begin{array}{c}\text { Before filter } \\
\text { (with fault) }\end{array}$ & 0.0071 & 0.0057 & 0.0040 & 0.0072 & 0.0056 & 0.0042 \\
\hline $\begin{array}{c}\text { After filter } \\
\text { (with fault) }\end{array}$ & 0.0001 & 0.0002 & 0.0004 & 0.0002 & 0.0002 & 0.0005 \\
\hline
\end{tabular}


After the signals being filtered, MAE is calculated to see the accuracy of the measured outputs and the RBF model outputs for both data samples. The MAE of the proposed RBF networks model can be summarized as in Table 2 . This table shows the MAE of the signals during the training and testing of free data faults for Samples1 and Samples2. Later, the RBF networks model is tested again, but now with the existing of faulty data occurred in the PEMFC systems. From the MAE measurements for both data, it shows that the MAE is high because it contains faulty data sets. The MAE of this faulty data is measured before it is filtered. Once the faulty data have been identified and detected, it shows that the MAE is also reduced. From the result obtained, the MAE after FD for NP is $0.0001, \lambda \mathrm{O}_{2}$ is 0.0002 and SV is 0.0004 for Samples1 while for Samples2, the MSE after the FD for NP is $0.0002, \lambda \mathrm{O}_{2}$ is 0.0002 and $\mathrm{SV}$ is 0.0005 .

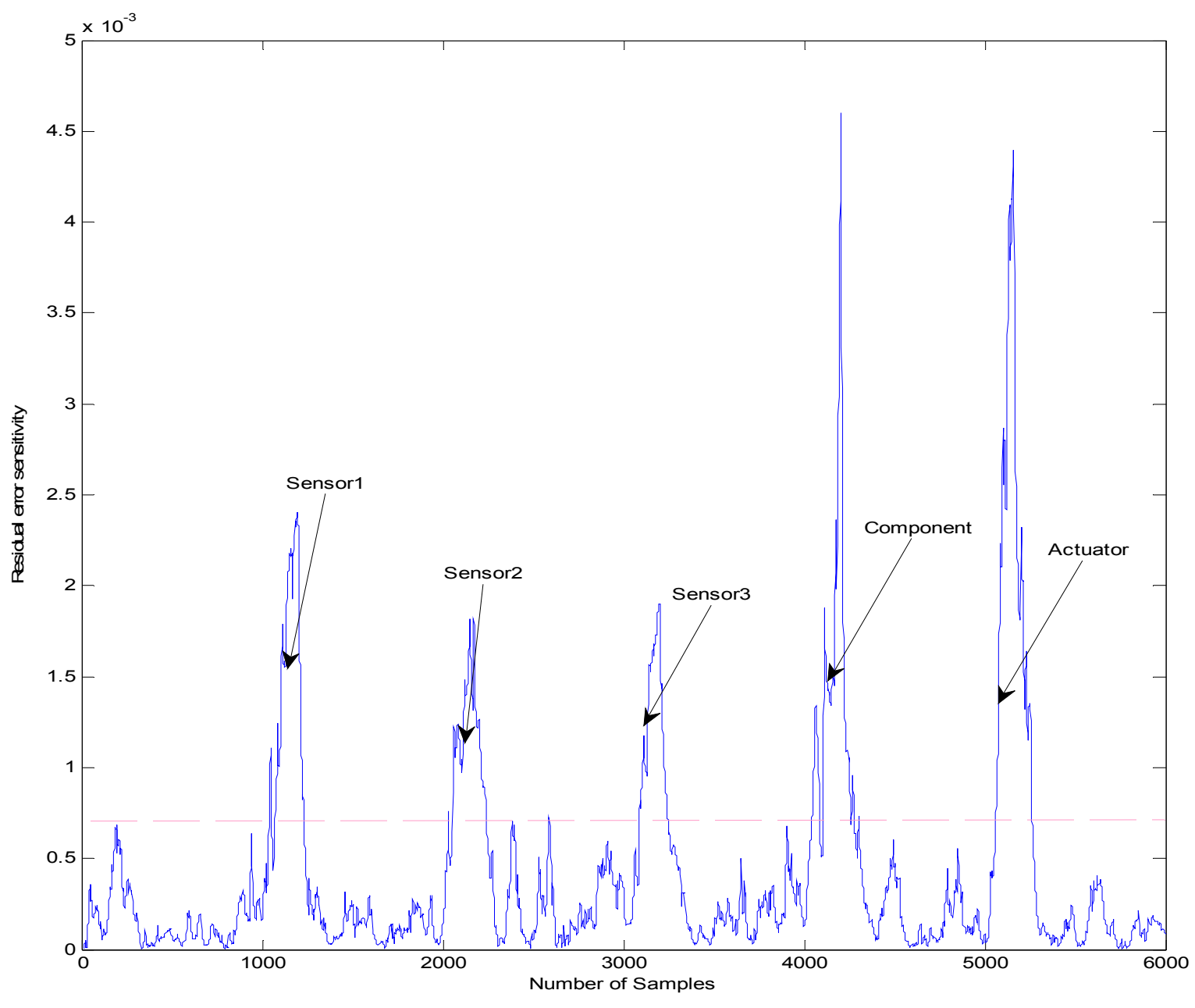

Figure 13: The fault detection of faulty signal of Samples1 and respective threshold

Figure 13 shows that the proposed model-based FD able to detect the five faults occur in the PEMFC systems after the implementing of residual errors sensitivity calculation. It shows that this proposed intelligent FD able to detect and locate the location of faults accurately. While in Figure 14 shows that final FD results obtained from the proposed model used for Samples2 data set. Again, it shows that this intelligent FD able to detect the five faults occurs in the system no matter where the faults are. The proposed intelligent FD method implement in this work have successfully detects all five faults in the PEMFC systems. 


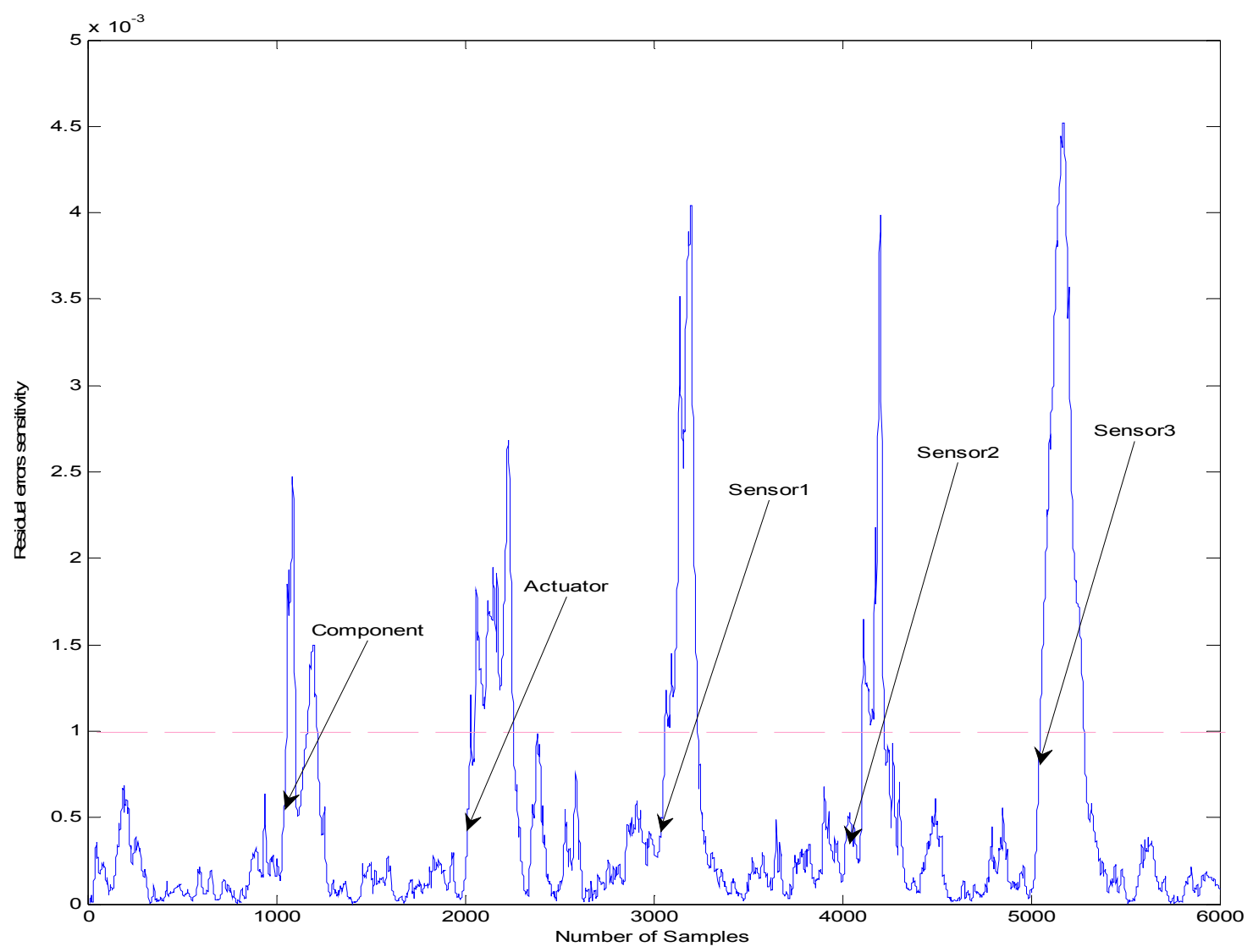

Figure 14: The fault detection of faulty signal of Samples2 and respective threshold

\section{Conclusions}

In this paper, a new model-based FD developed for PEMFC dynamic systems have been developed. The RBF networks model is used to do fault diagnosis which includes fault identification and classification. Here, the RBF networks has been developed to simulate free faults and faulty data sets and later implement FD to detect five faults happen inside the PEMFC dynamic systems. This intelligent FD monitoring systems is a model-based diagnosis method with residual errors sensitivity generation. It permits the detection of actuator, component and sensors faults. This intelligent FD can be implemented as a condition monitoring in the PEMFC to avoid oxygen starvation which later can damage the membrane and this overall can effects the performance of PEMFC. The result presented in this work showed that faults can clearly be detected by the proposed RBF networks algorithm. The proposed method for intelligent FD applied to PEMFC systems using the RBF networks shows a successful finding in order to detect all five faults in the PEMFC systems efficiently.

$\begin{array}{ll}\text { Nomenclature } & \\ \text { FD } & \text { Fault Detection } \\ \text { PEMFC } & \text { Proton Exchange Membrane Fuel Cell } \\ \text { RBF } & \text { Radial Basis Function } \\ \text { K2 } & \text { Bayesian-Score } \\ \text { MCMC } & \text { Markov Chain Monte Carlo } \\ \text { ENN } & \text { Elman Neural Network } \\ \text { FCS } & \text { Fuel Cell Stack } \\ \text { MAE } & \text { Mean Absolute Error } \\ \text { RAS } & \text { Random Amplitude Signals }\end{array}$




\section{References}

Albert.R., Ramon.S., Vicenc.P. and Teresa.E. 2007. Efficient Optimal Sensor Placement for Model-based FDI using an Incremental Algorithm. Proceedings of the $46^{\text {th }}$ IEEE Conference on Decision and Control, pp. 2590-2595.

Ari.I., Anna.G.S. and Denise.M. 2005. Model-based detection of hydrogen leaks in a fuel cell stack. Proceedings of the $44^{\text {th }}$

Conference on Decision and Control, pp. 1017-1022.

Carlos.A.R-P., Roberto.G., Luis. M-S., Jenny.R., Alfonso.R. and Giovanni.S. 2010. A PEM Fuel-Cell Model Featuring Oxygen-

Excess-Ratio Estimation and Power-Electronics Interaction. IEEE Transaction on Industrial Electronic, Vol. 57, pp. 1914-1924.

Jay.T.P., Huei.P. and Anna.G.S. 2004a. Control Fuel Cell Breathing. IEEE Control Systems Magazine, pp. 30-46.

Jay.T.P., Huei.P. and Anna.G.S. 2004b. Control-Oriented Modeling and Analysis for Automotive Fuel Cell Systems. Journal of Dynamics Systems, Measurements, and Control, Vol. 126, pp. 14-26.

Jay.T.P., Anna.G.S. and Huei.P. 2004c. Control of Fuel Cell Power Systems (Principles, Modeling, Analysis and Feedback Design). United State of America. Springer.

Jean-Pierre.G. and Joseph.K.K. 1995. Early detection and Continuous Monitoring of Dissolved Key Fault Gasses in Transformers and Shunt Reactors. Electrical Electronics Insulation Conference, pp. 285-293.

Luis.A.M.R., Marcelo.G.S. and Paulo.E.M. 2006. Fault identification in Fuel Cells Based on Bayesian Network Diagnosis. ABCM Symposium Series in Mechatronics, Vol. 2, pp. 757-764.

Mingyu.L, Songbai.H. and Xiaodong.L. 2009. Complex Radial Basis Function Networks Trained by QR-Decomposition Recursive

Least Square Algorithms Applied in Behavioral Modeling of Nonlinear Power Amplifiers. International Journal of RF and

Microwave Computer-Aided Engineering, pp. 634-646.

Mohamed.E.L. and Stephane.L. 2009. Identification and monitoring of PEM electrolyser based on dynamical modeling.

International Journal of Hydrogen Energy 34, pp. 5992-5999.

Roozbeh.R., Hadi.D., Vasile.P. and Caro.L. 2009. Model-based fault detection and isolation of a steam generator using neurofuzzy networks. Neurocomputing, Vol. 72, pp.2939-2951

Roy.A.G., Luis.A.M.R. and Paulo.E.M. 2008. Fault Diagnosis in Fuel Cells Based on Bayesian Networks. ABCM Symposium Series in Mechatronics, Vol. 3, pp. 424-433.

Salim.I. and Denver.C. 2009. Model-based estimation of PEM fuel-cell systems. $3^{\text {rd }}$ IEEE Multi-conference on Systems \& Control (MSC 2009), pp. 1397-1402.

Teresa.E., Diego.F., Salvado.D.L., Vicenc.P, Joseba.Q., Jordi.R. and Maria.S. 2009. Model-based fault diagnosis in PEM fuel cell systems. Journal of Power Sources 192, pp. 216-223.

Yousfi.S.N., Candusso.D., Hissel.D. and Mocoteguy.P. 2010. Model-based diagnosis for proton exchange membrane fuel cells. Mathematics and Computer In Simulation, pp. 158-170.

Zhai Y.J. and Dingli. Y. 2007. Radial Basis Function Feedback Control for Air Fuel Ratio of Spark Ignition. Proceedings of Institute Mechanical Engineers, Part D: J. Automobile Engineering, Vol. 22, pp. 415-428.

\section{Biographical notes}

Mahanijah Md Kamal received B.Eng. (Hons.) degree in Electrical Engineering from Institute Technology MARA Selangor, Malaysia in 1999 and M.Eng. degree in Communication and Computer from University Kebangsaan, Malaysia in 2005. She is currently a PhD student at Liverpool John Moores University, Liverpool, UK. Her research interest includes artificial intelligence, fault tolerant control, fault detection and isolation.

Prof Dingli Yu received B.Eng. degree from Harbin University of Civil Engineering, China, in 1982, the master degree from Jilin University of Technology (JUT), China, in 1986, and, and the PhD degree from Coventry University, UK, in 1995, all in electrical engineering. He was a lecturer at JUT from1986 to 1990 before he came to University of Salford as a visiting researcher in 1991. He then worked at Liverpool John Moores University as a post-doctoral researcher since 1995 and became a lecturer in 1998, where he is currently a professor of control systems. His research interests include fault detection and fault tolerant control of bilinear and nonlinear systems, adaptive neural networks and their control applications, and model predictive control for chemical processes and engine systems.

Received December 2010

Accepted April 2011

Final acceptance in revised form July 2011 